\title{
Reparación de una disección de aurícula izquierda. Presentación de un caso
}

\author{
Manuel Quiroz F. ${ }^{1,2}$, Minerva Jara C. ${ }^{2}$ y Manuel Novajas B. ${ }^{2,3}$
}

\section{Repair of left auricular dissection. Case report}

Introduction: Left atrial dissection is an infrequent but potentially fatal complication of cardiac surgery. It is frequently associated with mitral valve surgery, both its repair and replacement, with an incidence of $0.16 \%$. However, other procedures such as percutaneous interventions can also be predisposing factors. Objectives: To report the surgical resolution of a left atrial dissection case and contribute to the national casuistry. Materials and Method: Clinical, imaging and photographic record of the clinical episode. Results: A patient who underwent retrograde radiofrequency ablation during the post-interventional period with heart failure and whose study identifies an atrial dissection. Mitral ring repair, subvalvular apparatus repair and pericardial patch was performed, the patient evolves with favorable clinical and sonographic evolution. Discussion: The treatment of this entity should be analyzed case by case, the etiology related to percutaneous procedures is different to that caused by mitral valve surgery and this should be considered when choosing a therapeutic option. Conclusion: Repairing an atrial dissection with a patch is a good alternative in these cases.

Key words: left atrial dissection; mitral valve surgery; transoesophageal echocardiography.

\section{Resumen}

Introducción: La disección de la aurícula izquierda es una complicación infrecuente, pero potencialmente fatal de la cirugía cardíaca. Es frecuentemente asociada a cirugías de la válvula mitral, tanto su reparación el reemplazo, con una incidencia de $0,16 \%$. Sin embargo, otros procedimientos como intervenciones percutáneas también presentan este riesgo. Objetivos: Presentar la resolución quirúrgica de un caso de disección de aurícula izquierda y aportar a la casuística nacional. Materiales y Método: Registro clínico, imagenológico y fotográfico del episodio clínico. Resultados: Una paciente que fue sometida a ablación por radiofrecuencia por vía retrógrada, y cursa durante el periodo postintervencional con insuficiencia cardíaca y su estudio identifica una disección auricular. Se realiza reparación del anillo mitral, plastía del aparato subvalvular y parche de pericardio, la paciente presenta evolución clínica y ecográfica favorable. Discusión: El tratamiento de esta entidad debe analizarse caso a caso, ya que la etiología relacionada a procedimientos percutáneos es diferente a la causada por cirugía valvular mitral. Conclusión: La reparación de una disección auricular con parche es una buena alternativa de tratamiento en estos casos.

Palabras clave: disección de aurícula izquierda; cirugía valvular mitral; ecocardiografía transesofágica.

\section{Introducción}

La disección de la aurícula izquierda (AI) es una entidad infrecuente, la información disponible corresponde a casos clínicos en los cuales la etiología es diversa ${ }^{1-3}$.

Esta entidad tiene un amplio espectro de presentación, se asocia a complicaciones como insuficiencia mitral, arritmias y fenómenos embólicos. El sitio más común de la disección es la pared posterior.
La etiología puede dividirse en dos grupos principales; las causas quirúrgicas y las no quirúrgicas. Entre las causas quirúrgicas la más frecuente es la cirugía valvular mitral y entre las causas no quirúrgicas encontramos el trauma contuso, la endocarditis infecciosa y en los últimos años se han presentado casos posteriores a procedimientos percutáneos como la coronariografía y la ablación por radiofrecuencia ${ }^{4-6}$.

El manejo depende de la inestabilidad hemodi-
Universidad de Valparaíso. Valparaíso, Chile.

${ }^{2}$ Servicio de Cirugía Cardiovascular, Hospital Dr. Gustavo Fricke. Viña del Mar, Chile.

${ }^{3}$ Fundación Cardiovascular Dr. Jorge Kaplan Meyer. Viña del Mar, Valparaíso, Chile.

Recibido el 8 de julio de 2019 y aceptado para publicación el 29 de agosto de 2019.

Correspondencia a: Dr. Manuel Quiroz F. quirozflores.manuel@gmail. com 
námica y de la etiología, el uso de balón de contrapulsación intraaórtico contribuye a disminuir la postcarga al igual que la depleción de volumen. La cirugía debe corregir el defecto y depende principalmente de la etiología.

A continuación, presentamos un caso secundario a ablación por radiofrecuencia de una arritmia supraventricular y la técnica quirúrgica utilizada.

\section{Presentación del caso}

Paciente mujer de 55 años, con antecedentes médicos de taquicardia paroxística supra ventricular (TPSV) diagnosticada como taquicardia auricular perihoz en seno coronario que fue tratada con ablación por radiofrecuencia el año 2007.

La paciente se mantuvo en control ambulatorio sin incidentes durante 11 años, sin embargo, durante el último año presentó episodios aislados de palpitaciones y ansiedad, por lo cual consultó en varias oportunidades en atención primaria constatándose TPSV, siendo derivada a cardiología descartando causas valvulares o isquémicas, por lo que se decidió estudio electrofisiológico que se realizó el 7 de octubre de 2018. Durante el estudio se identificó taquicardia auricular a nivel del ostium del seno coronario y se aplicó radiofrecuencia. El mapeo por cateterismo arterial se realizó por acceso femoral izquierdo y se avanzó de forma retrógrada a través de la válvula aórtica a la aurícula izquierda (AI), evidenciando un foco en región paraseptal posterior, donde se aplicó radiofrecuencia logrando eliminar actividad ectópica.

El procedimiento concluyó sin incidentes, la paciente evolucionó en buenas condiciones, se decidió el alta a las $24 \mathrm{~h}$ del procedimiento.

La paciente consulta en el servicio de urgencias el 14 de octubre de 2018, por cuadro de dolor precordial EVA 8/10 de un día de evolución, asociado a disnea y náuseas. Además, refiere cefalea holocránea intensa.

El examen físico no muestra alteraciones agudas y el electrocardiograma identifica arritmia por fibrilación auricular con respuesta ventricular rápida por lo que se indica lanatocido $\mathrm{C}$ logrando ritmo sinusal y regresión de los síntomas. Se decidió alta y control ambulatorio.

Debido a nuevo episodio de dolor precordial de intensidad máxima de $20 \mathrm{~h}$ de evolución y disnea en reposo, la paciente consulta el 28 de octubre de 2018 en el servicio de urgencias, al examen físico destaca con apremio respiratorio, taquicardia y llene capilar retardado. En el examen físico destacaba soplo de insuficiencia mitral intensidad III/VI y murmullo pulmonar disminuido en las bases.

Se realiza electrocardiograma que evidencia ritmo sinusal, se solicitan exámenes, destacando leucocitosis 19.000 células/uL, dímero D 2728, la radiografía de tórax evidenció congestión bilateral e imagen sugerente de condensación en lóbulo inferior derecho.

Se decidió hospitalización para continuar estudio, se descartó tromboembolismo pulmonar mediante angiotc, se inició tratamiento antibiótico de amplio espectro sospechando una neumonía con criterios de gravedad.

Debido a la persistencia de disnea y signos de congestión cardíaca se solicita ecocardiografía, en la que destacó AI dilatada, en relación al anillo mitral, el velo mitral posterior se encontró ampliamente desprendido, en continuidad con disección que ocupa la cavidad auricular izquierda determinando insuficiencia mitral severa excéntrica, reflujo tricuspídeo e hipertensión pulmonar severa (Figuras 1-3).

Con estos antecedentes se hospitaliza en Unidad de Cuidados Intensivos Cardiovasculares con diagnóstico de shock cardiogénico secundario a insuficiencia mitral aguda y disección auricular izquierda.

Se presenta caso a equipo quirúrgico y se decide reparación quirúrgica, la paciente ingresa a pabellón el 30 de octubre de 2018; se realiza bajo circulación extracorpórea mediante canulación aórtica, canulación venosa bi-cava y cardioplegia anterógrada in-

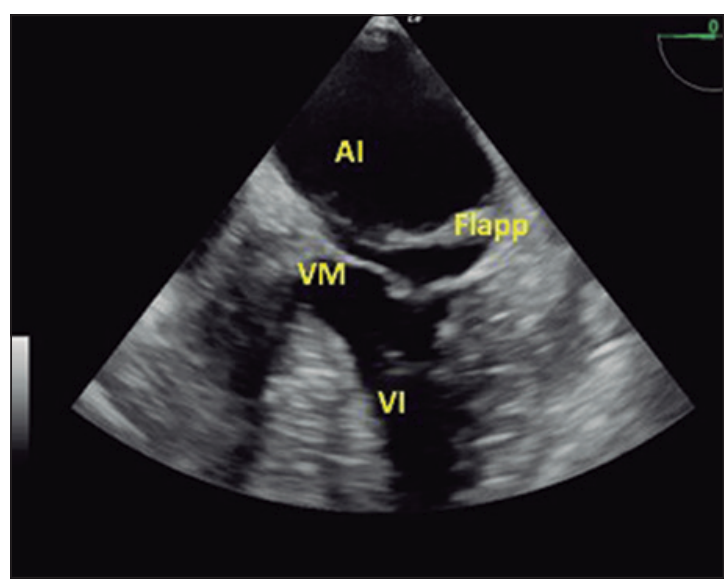

Figura 1. ETE pre operatorio. Vista en cuatro cámaras: En la cavidad de la aurícula izquierda se observa un flapp de disección que va desde el septum interauricular a la pared lateral de la aurícula izquierda. Al: Aurícula izquierda. VI: Ventrículo izquierdo. VM: Válvula mitral. 


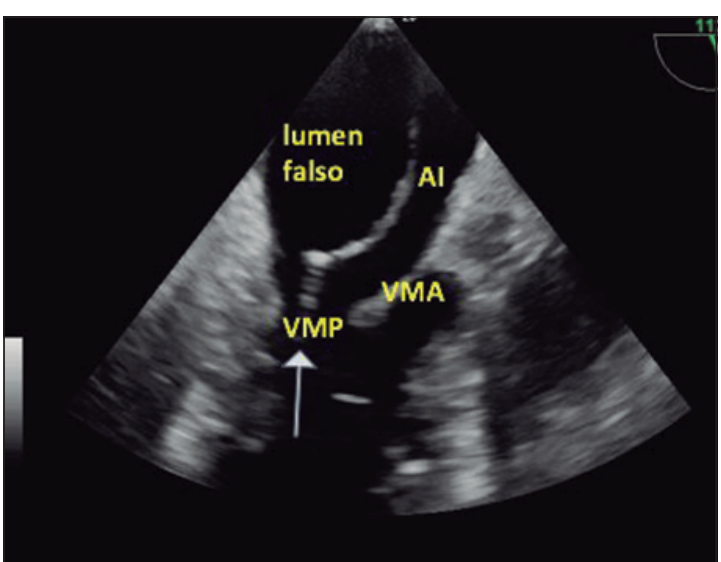

Figura 2. ETE preoperatorio. Vista de dos cámaras: Se observa solución de continuidad (flecha) que comunica la cavidad del ventrículo izquierdo con el lumen falso de la cavidad auricular. Al: Aurícula izquierda. VI: Ventrículo izquierdo. VMP: Velo mitral posterior. VMA: Velo mitral anterior.

termitente, abordaje transeptal evidenciando amplio flapp de disección de la pared atrial en relación con el velo posterior mitral, además de una solución de continuidad del reborde de la válvula correspondiente a $\mathrm{P} 3$, de $3 \mathrm{~cm}$ de extensión, que alimenta el área disecada con flujo activo (Figura 4).

Se realiza reparación del anillo mitral con plastía de P3 mediante puntos de Ethibond 2.0 con pledget, más cierre de flapp de endocardio auricular con prolene 6.0 y se refuerza con parche de pericardio. Tiempo de circulación extracorpórea de 159 min y clamp 123 min (Figura 5).

La paciente cursa el postoperatorio inmediato con apoyo de balón de contrapulsación intraaórtico y drogas vasoactivas que se retiran de forma precoz.

La ecografía del posoperatorio inmediato evidencia el área de disección auricular izquierda trombosada, con presencia de venas pulmonares permeables y válvula mitral de configuración y función normal.

A las 24 h de evolución el lumen falso se observa trombosado sin flujo en su interior.

Se discute caso en reunión clínica y se define iniciar tratamiento anticoagulante y completar 3 meses de amiodarona antes de una nueva reevaluación debido a fibrilación auricular durante su estadía postoperatoria.

Al control ambulatorio la paciente se encuentra en buenas condiciones generales, asintomática y su última ecografía de control muestra disminución del área disecada con un trombo pequeño sin actividad en su interior (Figura 6).

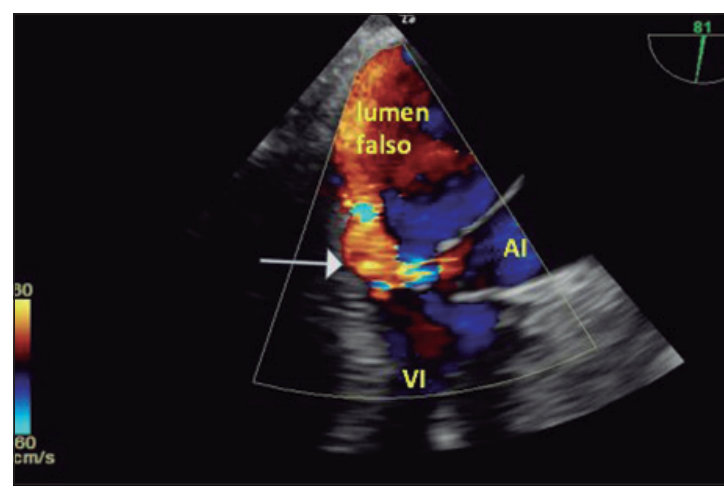

Figura 3. ETE preoperatorio. Vista de dos cámaras con doppler color: Se observa un yet de insuficiencia mitral severa excéntrico (flecha) desde el ventrículo izquierdo al falso lumen de la aurícula izquierda. Al: Aurícula izquierda. Vl: Ventrículo izquierdo.

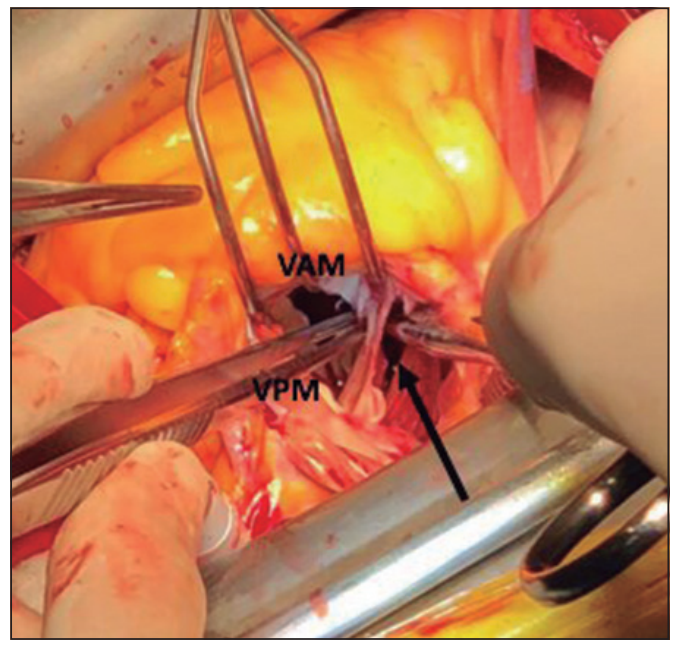

Figura 4. Visión transeptal de la aurícula izquierda. Se observa válvula mitral con evidente compromiso de P3 y flapp de disección que se origina en unión aurículo- ventricular y genera un lumen falso. VAM: Velo anterior mitral. VPM: Velo posterior mitral. Flecha: Lumen falso.

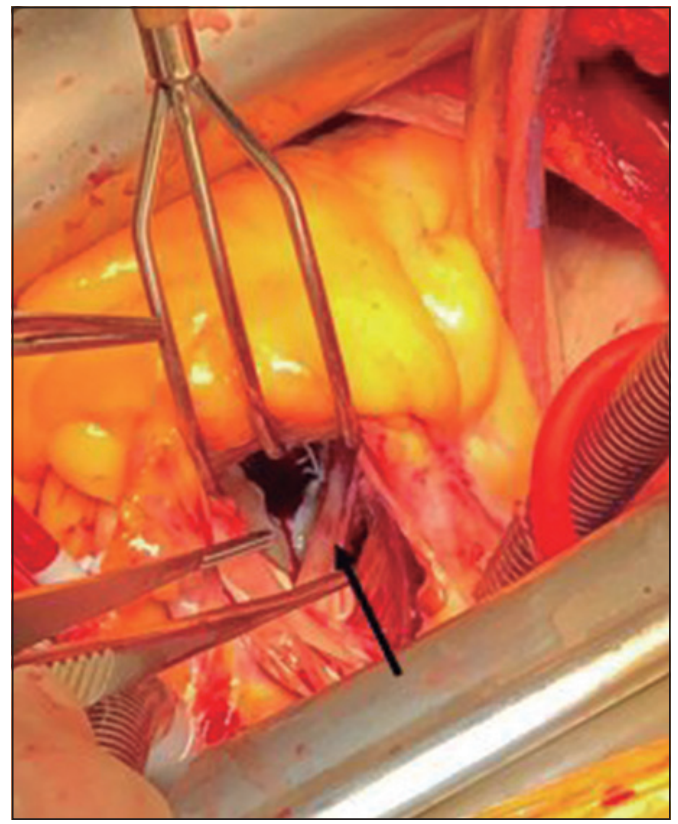

Figura 5. Visión transeptal de la aurícula izquierda. Flecha: Parche de pericardio en posición previo a la reparación. 
Figura 6. ETE 2 meses postoperatorio. Vista de eje largo. Se observa franca disminución del tamaño del trombo en la aurícula izquierda, sin efecto de masa intraluminal. Sin insuficiencia mitral (no documentado en esta imagen). VPM: Velo posterior mitral. VAM: Velo anterior mitral VI: Ventrículo izquierdo.

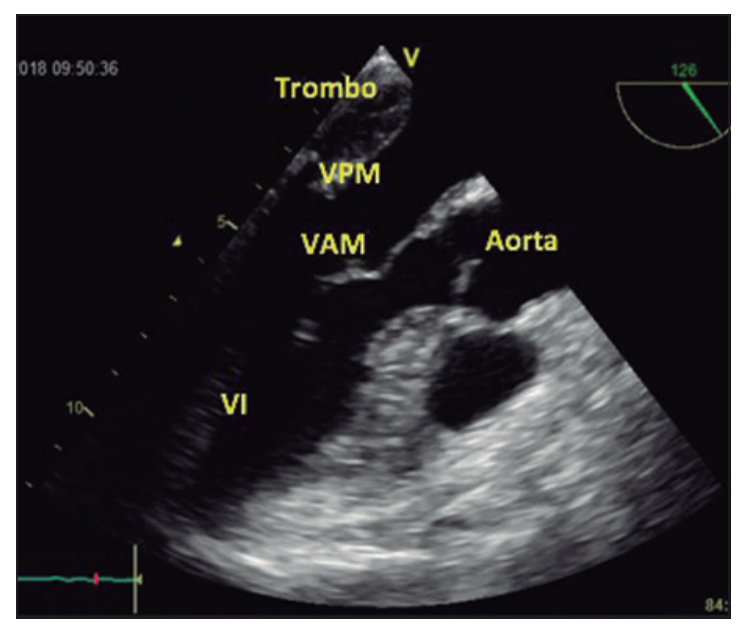

\section{Discusión}

La causa más frecuente es la cirugía de la válvula mitral, independiente del tipo de cirugía, reparación o reemplazo o del tipo de válvula protésica, Fukuhara et al. ${ }^{7}$, encontraron 87 casos reportados entre 1979 y 2014 , de los cuales el $56 \%$ correspondió a esta causa, mientras que la ablación por radiofrecuencia correspondió al $8 \%$ de los casos y la angioplastía fue un $6,9 \%$.

En ambos casos la causa es la manipulación de guías metálicas rígidas.

En nuestro caso, correspondió a una disección posterior a ablación con radiofrecuencia que incluyó el velo posterior de la válvula mitral, por lo tanto, planteaba dos problemas de causa no quirúrgica con gran compromiso hemodinámico.

La afectación de la pared posterior de la aurícula es más frecuente $80 \%$ de los casos, lo que puede explicarse porque los tejidos de fijación del velo posterior al anillo son principalmente musculares, con escaso tejido fibroso, mientras que hacia el velo anterior la fijación es más firme, presenta más tejido fibroso y muchas veces incluye calcificaciones.

El pronóstico de esta entidad depende de la presentación y el tipo de manejo, si el paciente se mantiene hemodinámicamente estable es posible realizar un manejo conservador con buen resultado, como observó Fukuhara en el $24 \%$ de los casos, esto supone el remodelamiento y la regresión del hematoma?.

Si el paciente se presenta inestable, la alternativa quirúrgica es la opción de tratamiento más segura con una mortalidad cercana al $13 \%$ de los casos ${ }^{7,8}$.

El diagnóstico se establece mediante criterios clínicos, hemodinámicos y ecocardiográficos.

La ecocardiografía transesofágica (ETE) es considerada el recurso diagnóstico de elección para evaluar aurícula izquierda, la anatomía del septo interauricular y la función de las válvulas nativas y protésicas. Debido a lo anterior es la alternativa para el diagnóstico de esta entidad independientemente de la causa desencadenante? .

Se puede realizar manejo conservador dependiendo de la condición clínica del paciente, sin embargo si el caso requiere de cirugía existen dos formas de abordar el problema, el cierre del defecto, que se puede realizar de forma primaria o con parche de pericardio o la derivación, en la cual se deriva el flujo desde el falso lumen hacia la aurícula derecha logrando descomprimir la aurícula izquierda ${ }^{10,11}$.

En los casos en los que se ve involucrada la válvula mitral, una alternativa es el reemplazo valvular. En nuestro caso, la reparación fue posible, ya que la etiología no fue cirugía valvular, y el defecto pudo ser reparado mediante sutura para cerrar el defecto disecante y el refuerzo con parche de pericardio.

\section{Conclusiones}

La disección de aurícula izquierda es una entidad infrecuente, su etiología y presentación son variables.

La sospecha principal debe ser ante procedimientos quirúrgicos o percutáneos y su manejo dependerá de la estabilidad hemodinámica y de la causa.

$\mathrm{Su}$ tratamiento precoz presenta una buena respuesta clínica, sin embargo, la cirugía puede ser compleja y desafiante, por lo que cada caso debe ser evaluado por un equipo experimentado.

\section{Responsabilidades éticas}

Protección de personas y animales. Los autores declaran que para esta investigación no se han realizado experimentos en seres humanos ni en animales.

Confidencialidad de los datos. Los autores declaran que en este artículo no aparecen datos de pacientes.

Conflictos de interés: no hay. 


\section{Bibliografía}

1. Maeda K, Yamashita C, Shida T, Okada M, Nakamura K. Successful surgical treatment of dissecting left atrial aneurysm after mitral valve replacement. Ann Thorac Surg. 1985;39:382-4.

2. Chida K, Ohkawa S, Nagashima K, Imai T, Kuboki K, Maeda S, et al. An autopsy case of incomplete left atrial rupture following left atrial infarction associated with left ventricular myocardial infarction. Jpn Circ J. 1995;59:299-302.

3. Schulte HD, Gramsch Zabel H, Hortskotte D, Losse B. Uncommon type of mitral insufficiency caused by perivalvular communications between left ventricle and left atrium. Thorac Cardiovasc Surg. 1990;38:33-5.
4. Cresce GD, Peluso D, Panfili M, Favaro A, Cannarella A, Picichè M, et al. Left atrial wall hematoma as a consequence of percutaneous coronary angioplasty. Ann Thorac Surg. 2012;93:e57-9.

5. Solzbach U, Beuter M, Haas H. Left atrial intramural hematoma after percutaneous coronary intervention. Int J Cardiol. 2010;141:e37-8.

6. Uçar O, Ciçekçioglu H, Diker E, Aydogdu $\mathrm{S}$. A rare complication of radiofrequency catheter ablation of left atrial tachycardia: atrial septal dissection and left atrial hematoma formation. Turk Kardiyol Dern Ars. 2010;38:279-81.

7. Fukuhara S, Dimitrova KR, Geller CM, Hoffman DM, Tranbaugh RF. Left atrial dissection: an almost unknown entity. Interact Cardiovasc Thorac Surg. 2015;20: 96-100.
8. Fukuhara S, Dimitrova KR, Geller CM, Hoffman DM, Ko W, Tranbaugh RF. Left atrial dissection: etiology and treatment. Ann Thorac Surg. 2013;95:1557-62.

9. Pérez-de Isla L, García MA, Moreno M, Bermejo J, Moreno R, López E, et al. Eficacia y seguridad de la ecocardiografía transesofágica en la fase aguda del infarto. Rev Esp Cardiol. 2002;55:1132-6.

10. Zamorano J, Moreno R, Almeria C, Serra V, Rodrigo J, Sánchez L. Rotura de la pared libre del ventrículo izquierdo durante prueba de estrés con dobutamina. Rev Esp Cardiol. 2002;55:312-4.

11. Gallego P, Oliver JM, González A, Domínguez FJ, Sánchez A, Mesa J. Left atrial dissection: pathogenesis, clinical course, and transesophageal echocardiographic recognition. J Am Soc Echocardiogr. 2001;14:813-20. 